\title{
Nanometre-scale infrared chemical imaging of organic matter in ultra-carbonaceous Antarctic micrometeorites (UCAMMs)
}

\author{
Jérémie Mathurin ${ }^{1}$, Emmanuel Dartois ${ }^{2}$, Thomas Pino ${ }^{2}$, Cécile Engrand ${ }^{3}$, Jean Duprat ${ }^{3}$, \\ Ariane Deniset-Besseau ${ }^{1}$, Ferenc Borondics ${ }^{4}$, Christophe Sandt ${ }^{4}$, and Alexandre Dazzi ${ }^{1}$ \\ ${ }^{1}$ Laboratoire de Chimie Physique, CNRS, Université Paris Sud, Université Paris-Saclay, 91405 Orsay Cedex, France \\ e-mail: jeremie.mathurin@u-psud.fr \\ ${ }^{2}$ Institut des sciences moléculaires d'Orsay, CNRS, Université Paris Sud, Université Paris-Saclay, 91405 Orsay, France \\ ${ }^{3}$ Centre de Sciences Nucléaires et de Sciences de la Matière (CSNSM), CNRS/IN2P3, Université Paris Sud, Université Paris-Saclay, \\ 91405 Orsay, France \\ ${ }^{4}$ Synchrotron Soleil, L'Orme des Merisiers, BP 48 Saint Aubin, 91192 Gif-sur-Yvette Cedex, France
}

Received 26 July 2018 / Accepted 12 December 2018

\begin{abstract}
Aims. The composition of comets and asteroids sheds light on the formation and early evolution of the solar system. The study of micrometeorites containing large concentrations of carbonaceous material (i.e. ultra-carbonaceous antarctic micrometeorites, UCAMMs) allows for unique information on the association of minerals and organics at surface of icy objects (comets) to be obtained. Methods. In this work we map the organic matter of UCAMMs collected in the Antarctic snow, at sub-wavelength spatial scales using the Atomic Force Microscope InfraRed (AFMIR) technique. The sample preparation did not involve any chemical pretreatment to extract organic matter. The AFMIR measurements were performed on a limited spectral coverage $\left(1900-1350 \mathrm{~cm}^{-1}\right)$ allowing chemical functional groups to be imaged at spatial scales relevant to the study of micrometeorites.

Results. The AFMIR images reveal the variability of the functional groups at very small scales and the intimate association of carbonand oxygen-bearing chemical bonds. We demonstrate the possibility to potentially separate the olefinic and aromatic $\mathrm{C}=\mathrm{C}$ bonding in the subcomponents of the UCAMM fragment. These variations probably originate in the early mixing of the different reservoirs of organic matter constituting these dust particles. The measurements demonstrate the potential for analysing such complex organicmatter - mineral association at scales below the diffraction limit. The development of such studies and extension to the full infrared range spectral coverage will drive a new view on the vibrational infrared analysis of interplanetary material.
\end{abstract}

Key words. astrochemistry - comets: general - meteorites, meteors, meteoroids - techniques: imaging spectroscopy methods: laboratory: solid state - interplanetary medium

\section{Introduction}

Primitive extraterrestrial objects witnessed the nascent protoplanetary disc around the young Sun, 4.5 billion years ago. Analyses of micrometeorites collected in Antarctica provide information on the origins of the Solar System and on disc evolution. Micrometeorites originating from the outer regions of our Solar System have recorded processes involved in the formation and evolution of icy bodies from the cometary reservoir. The underlying nature of the chemical bonds of the extraterrestrial material, and in particular the organic component, can be studied using infrared (IR) vibrational spectroscopy. This classic technique is limited by diffraction, with typical spot sizes sampling a range of a few micrometres in the mid-IR. It provides insights on the dust grain chemical structure on scales of a few microns, which is however much larger than the fine structures observed by other means such as NanoSIMS (with a spatial resolution of $\sim 100 \mathrm{~nm}$ ), scanning or transmission electron microscopy, or $\mathrm{X}$-ray absorption near edge spectroscopy (XANES) coupled to scanning microscopy (STXM). The STXM-XANES technique is powerful when the samples can be prepared in thin films, but some functional groups cannot always be fully separated due to the close proximity of certain resonances (e.g. Alleon et al. 2017). Coordination groups can however be grouped into several families for the analysis providing semi-quantitative to quantitative information depending on the response overlap of the functional groups under consideration. Recent progress has been achieved to overcome the inherent IR diffraction limit issue, by a savvy design coupling an atomic force microscope (AFM) to an IR laser illumination of the samples, giving an access to IR analytical capabilities at sub-wavelength spatial resolutions (e.g. Dazzi \& Prater 2017; Dazzi et al. 2012). This AFMIR technique allows for a fragment of UCAMMs to be mapped without pre-treatment, and for the different forms of its organic matter to be quantitatively traced; it complements and is sensitive to the functional group information gained with other techniques. In this work we apply the AFMIR technique to characterise the variations in the chemical bond of organic matter in UCAMMs with sub-wavelength spatial resolution. We show that resolution at this scale is essential to understanding the diversity of chemical functional groups in such complex primitive samples.

\section{Organic matter in Antarctic micrometeorites}

Interplanetary dust particles from asteroids or comets can be characterised in situ or returned to Earth by space probes. The Stardust and Hayabusa spatial missions returned samples from comet Wild2 and asteroid Itokawa, respectively. Small interplanetary dusts are also collected from the stratosphere by 
NASA aeroplanes, while the main mass of extraterrestrial dust surviving atmospheric entry as particles is recovered at Earth surface (e.g. from collections in the polar caps). Antarctica represents an optimal environment for their preservation, minimising their terrestrial weathering after they have fallen. In this paper we present analyses of micrometeorites recovered from central Antarctic snow in the vicinity of the CONCORDIA station (e.g. Duprat et al. 2007).

Collections of micrometeorites are performed in several locations in Antarctica and most of the particles recovered contain only a few weight percent of carbon (e.g. Engrand \& Maurette 1998; Taylor et al. 2008; van Ginneken et al. 2012). The so-called ultra-carbonaceous Antarctic micrometeorites (UCAMMs) contain extremely high concentrations of carbonaceous matter (Nakamura et al. 2005; Duprat et al. 2010; Yabuta et al. 2012, 2017) and represent about $1 \%$ of the micrometeorites in the CONCORDIA collection (Dobrica et al. 2010). The mineralogical, chemical and isotopic characteristics of UCAMMs indicate that they most probably originate from the surface of icy parent bodies (Dartois et al. 2013, 2018; Bardin et al. 2014; Augé et al. 2016; Charon et al. 2017). The high organic concentration in UCAMMs allows direct analyses to be made without the pre-treatment generally applied to extract organic matter from meteorites.

Effort has been made to overcome the diffraction limitations imposed by classical Fourier transform IR microspectroscopy analyses (Kebukawa et al. 2010; Dominguez et al. 2014; Yesiltas \& Kebukawa 2016), and preserve the information on chemical functional groups that is complementary to conventional elemental and isotopic mapping provided by electron microscopy and secondary ion mass spectrometry (NanoSIMS). AFMIR provides the spatial resolution mapping capability and is also, if well calibrated, among the best near-field quantitative techniques (Ramer et al. 2017).

\subsection{Sample preparation}

The fragments analysed in this study were collected during the 2006 (DC06-07-18-b) and 2016 (DC16-14-309) Antarctic CONCORDIA collection campaigns. The DC06-07-18-b sample is a fragment of UCAMM DC06-07-18 previously analysed using IR and Raman microscopy (Dartois et al. 2018). This sample was prepared for analysis by pressing it between two diamond windows in order to obtain a flat and thin sample. These diamond substrates are $0.5 \mathrm{~mm}$ thick optical grade CVD diamond windows of $8 \mathrm{~mm}$ diameter, purchased from Element $\mathrm{Six}^{1}$. They are maintained in a counterbore made in a $20 \mathrm{~mm}$ diameter stainless steel holder with a central hole of $5 \mathrm{~mm}$ to allow for classical IR spectroscopy measurements at large scale before and after AFMIR measurements. The window holding the largest sample after pressing was analysed by AFMIR. Several analysis were made using this first preparation method, with progressively smaller thickness of the sample (upon pressing), allowing to determine the optimal sample thickness required for a good signal/noise ratio $(\mathrm{S} / \mathrm{N})$ in the AFMIR spectra. Once the optimal thickness was evaluated, the second sample (the DC16-14-309 fragment) was prepared by ultramicrotomy after embedding in sulphur, providing a more homogenous and flat surface. A drop of melted sulphur was applied to the UCAMM fragment and microtomed. The approximately micron-thick slices were deposited on a diamond substrate. Sulphur was then sublimated by gently warming the preparation to $50^{\circ} \mathrm{C}$ before analysis.

https://www.e6.com
The identification of the samples as UCAMMs is performed by analysing fragments of the same particle using Scanning Electron Microscopy equipped with Energy-Dispersive X-ray spectroscopy (SEM/EDX) and by isotopic measurements. We performed analyses on different fragments of the same particle to prevent contamination and/or modifications of the sample before AFMIR analysis. In addition, the non-destructive far-field IR spectrum recorded on a classical IR microscope of the fragment measured by AFMIR confirmed that it was of the same nature as the fragments analysed previously.

\subsection{Nanoscale IR spectroscopy (AFMIR)}

In this study nanoscale IR spectroscopy is achieved using the AFMIR technique which combines an IR tunable laser with an AFM. The detection is based on the photo-thermal expansion of the sample resulting from the IR absorption. This expansion is detected using an AFM probe. As the expansion is fast, it acts as a shock for the AFM cantilever leading it to oscillate at its contact resonant frequencies. The amplitude of the generated oscillations is proportional to the absorbance of the sample directly under the AFM tip. In this situation, the spatial resolution is not limited by the diffraction limit as is the case for classic IR microscopy. The spatial resolution of AFMIR is directly correlated to the AFM tip radius which theoretically allows for nanometer-sized resolution to be reached.

The system used in this study is a NanoIR2s from Anasys Instrument. In this setup the IR beam is focused from the top side of the sample onto the AFM cantilever. An Au-coated silicon tip is used to avoid artefact effects linked to the IR absorption of silicon. The system is coupled to a multi-chip quantum cascade laser (QCL) source that covers a portion of the mid-IR range $\left(1900-1550 \mathrm{~cm}^{-1}\right.$ for the first sample and extended to $1900-1350 \mathrm{~cm}^{-1}$ for the second sample). An advantage of the QCL source is the tunability of the repetition rate which allows different AFMIR analysis modes. The first sample (DC06-07-18-b) was studied with AFMIR in contact mode (Fig. 1, left panel). In this case the repetition rate is chosen in order to match the contact resonant frequency of the AFM cantilever. As a consequence, the cantilever oscillations moved to an enhanced mode leading to an increase of the spatial resolution of the technique which can reach ten nanometers ( $\mathrm{Lu} \&$ Belkin 2011). For the second sample (DC16-14-309), the study was made using a novel acquisition mode: tapping AFM (Fig. 1, right panel). In this mode, the measurement is based on principles from heterodyne or multifrequency AFM in which multiple flexural eigenmodes of the AFM cantilever are excited or measured to enhance the sensitivity of the probe-sample interaction (Mathurin et al. 2018). In addition to the classic AFM measurement, we acquired local IR spectra (on fixed spots) for both samples by tuning the laser with a $2 \mathrm{~cm}^{-1}$ step. We performed acquisitions fixing the laser to a given wavenumber and observing the cantilever ringdown at each point, to obtain the AFM image (either in contact mode or in tapping mode) and the absorption signal map at this wavenumber simultaneously. The UCAMM sample DC06-07-18-b was analysed with the AFMIR nanoIR2 instrument in the $1900-1550 \mathrm{~cm}^{-1}$ range, covering the $\mathrm{C}=\mathrm{O}$ and $\mathrm{C}=\mathrm{C} / \mathrm{C}=\mathrm{N}$ stretching modes. We use the same configuration as in Lu et al. (2014). These authors have calculated that using their laser at $100 \%$ power, with $160 \mathrm{~ns}$ pulses, they estimate an increase of their sample temperature by $\Delta T<6 \mathrm{~K}$. We used even shorter pulses (120 ns) and laser power below $50 \%$ of the full power in tapping mode and below $10 \%$ in contact mode. We also performed test measurements under the 


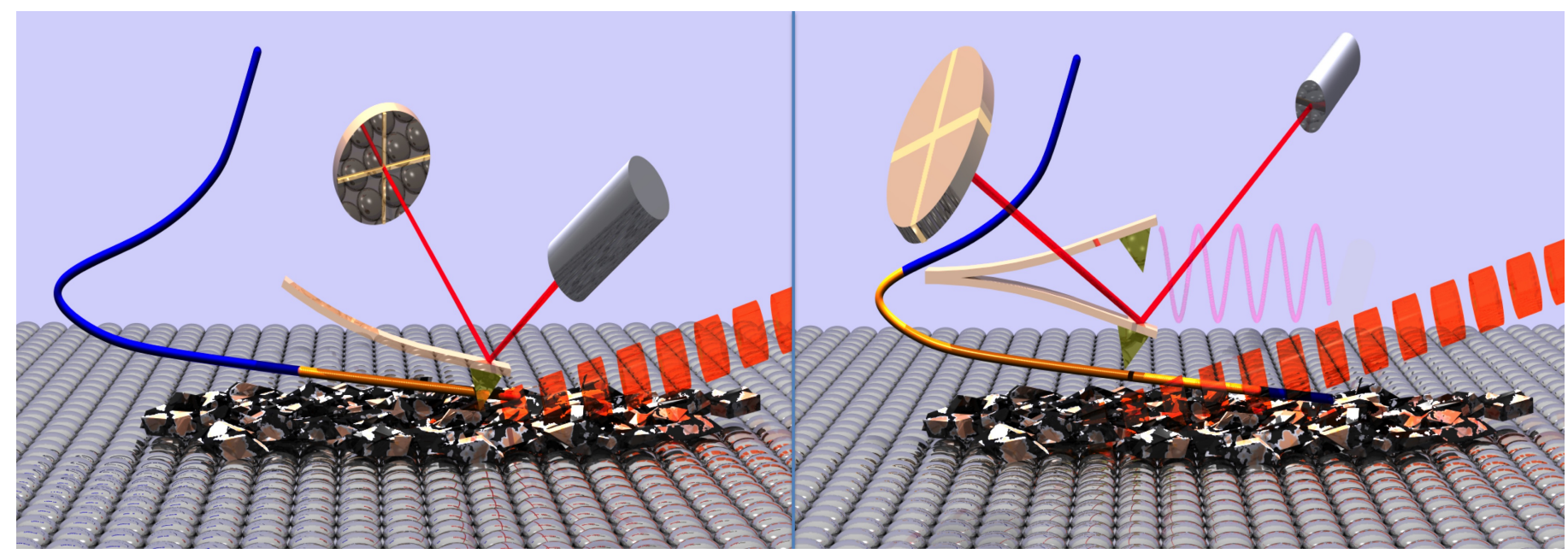

Fig. 1. Schematic view of the AFMIR contact (left panel) and tapping (right panel) modes. The interaction potential normal to the surface is illustrated (blue line). The approximate excursion ranges on this interaction potential for the two AFM modes are shown in yellow. In contact mode, the tip of the cantilever probe is in close contact with the surface and explores mainly the repulsive potential. In tapping mode, the cantilever oscillates and approaches the surface only for a short time, oscillating back and forth on the potential curve.

same conditions on hydrogenated amorphous carbon synthetic samples that we generally use to reproduce interstellar hydrogenated carbons observed in the diffuse interstellar medium (e.g. Dartois et al. 2017), with no measurable structural or chemical changes before or after AFMIR measurements. Moreover, the fact that far-field FTIR spectra recorded after AFMIR measurements for the analysed samples are typical of the considered UCAMMs fragments, showing no pattern of laser damage, confirms that no damage was done to the samples using such low power and short pulse lengths. Signs of stripping, a well-known issue in AFM measurement, were detected by convolving the image with a Laplacian filter and detecting the points above $3 \sigma$ in the result. These points representing a few percent of the map are therefore flagged and interpolated with a bilinear interpolation algorithm. The maps are also offset corrected using background pixels as reference. All maps were subsequently noise-filtered applying an adaptive filter estimating the local standard deviation over an area of five pixels. The intensity maps were divided by height measured by the AFM to display the normalized (i.e. thickness-independent) intensity. To avoid division by zero, the maps were multiplied by a mask discarding pixels in the background and immediate contours of the samples. The intensity scales are displayed in arbitrary units but using the same scales for each image set for each sample. Prior to calculating the ratios of the AFMIR maps, we computed the $2 \mathrm{D}$ cross-correlation function of the two image intensities of the maps to readjust the small pixel spatial shift mismatch of the $1710 \mathrm{~cm}^{-1}$ image relative to the $1600 \mathrm{~cm}^{-1}$ one. The highest spatial resolutions achievable in the contact and tapping modes with the cantilevers used are about 20 and $30 \mathrm{~nm}$, respectively. For the DC06-07-18-b measurements the maps were slightly smoothed with respect to the initial spatial resolution (and sampling) by applying a noise filter, with a kernel corresponding to a spatial resolution of about $200 \mathrm{~nm}$ (regions A) and $100 \mathrm{~nm}$ (region B). For all maps, the ratios are displayed only for pixels with intensities above $3 \sigma$ prior to the division (pixels below $3 \sigma$ were set to background color). After the AFMIR measurements, a far-field global IR transmission spectrum was recorded for comparison using a Nicolet Continuum II IR microscope equipped with an MCT detector, using the internal thermal source, at a spectral resolution of $2-4 \mathrm{~cm}^{-1}$. An aperture of $5 \times 10$ to $10 \times 10 \mu \mathrm{m}$ was used to cover the main portion of the UCAMMs fragments.

\subsection{SEM and EDX}

After the AFMIR measurements were performed, the samples were observed by scanning electron microscopy (in secondary and backscattered electron mode), and analysed by energy dispersive X-ray spectroscopy (SEM/EDX at 12-15kV). Backscattered electron (BSE) images of fragments of both UCAMMs are displayed in Fig. 2 for DC06-07-18-b and Figs. 6 and 8 for DC16-14-309. The backscattered electron imaging provides a chemical contrast of the sample, with the minerals appearing brighter than the purely organic component. Energy-dispersive $\mathrm{X}$-ray maps of sulphur, silicon, iron (silicates and Fe-Ni sulphides), magnesium, and oxygen were acquired and are shown hereafter in selected areas. For DC16-14-309, EDX sulphur maps show that in very localised regions, the embedding sulphur matrix has not fully evaporated. Such regions can be identified since in extraterrestrial matter sulphur is generally associated with iron, and a counterpart in the iron EDX map is therefore expected.

\section{Results and discussion}

\section{1. $D C 06-07-18-b$}

The two (overlapping) selected regions of UCAMM DC06-0718 -b measured with the AFMIR are shown in Fig. 2 on a backscattered electron image. The regions $\mathrm{A}(20 \times 10 \mu \mathrm{m})$ and B $(10 \times 5 \mu \mathrm{m})$ were analysed with steps of 20 and $10 \mathrm{~nm}$, respectively. The far-field global Fourier transform IR transmission spectrum recorded with an aperture of $10 \times 10$ microns and covering the majority of the micrometeorite is shown in Fig. 3. The functional groups identified by FTIR in UCAMMs are presented in Dartois et al. (2018). In particular, the far-field IR spectrum of Fig. 3 is similar to that of thin laboratory-synthesised, hydrogenated carbon nitride films presented in Fig. 5 of Dartois et al. (2013). The absorption band peaking around $1600 \mathrm{~cm}^{-1}$ can be attributed to $\mathrm{C}=\mathrm{C}$ and $\mathrm{C}=\mathrm{N}$ bonds from the nitrogen-rich 

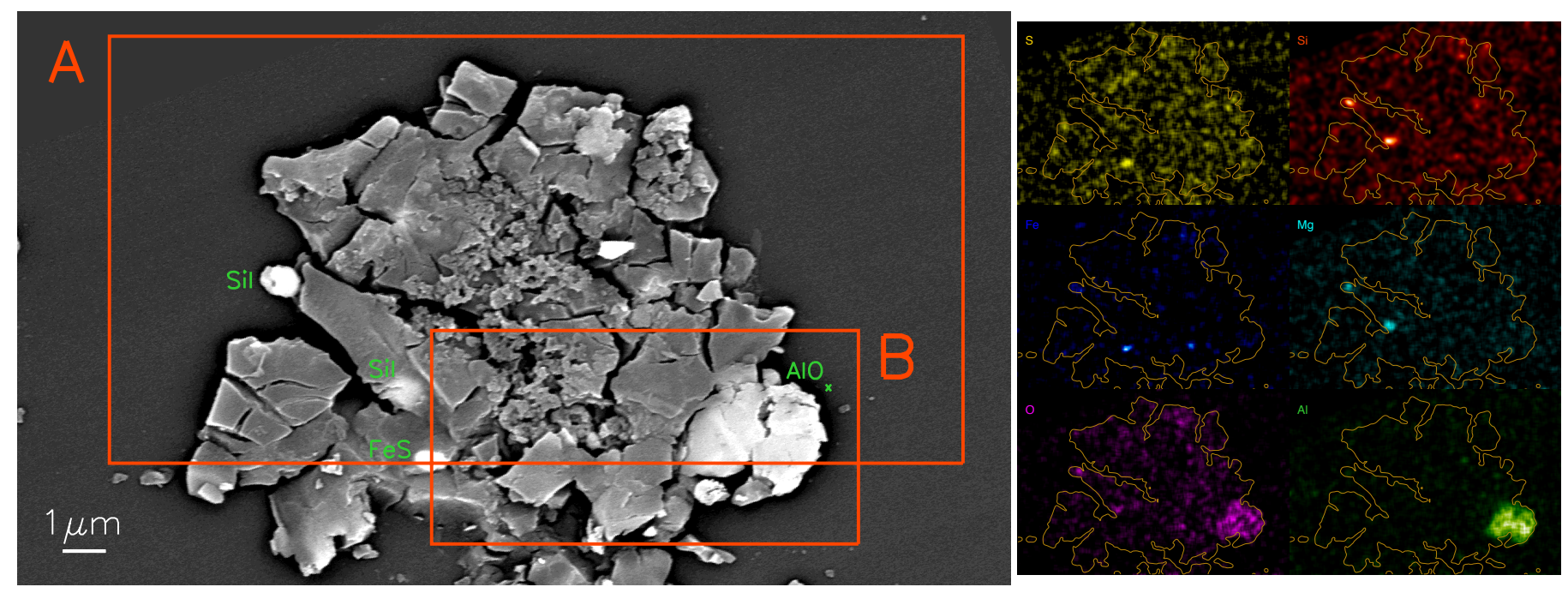

Fig. 2. Left: back-scattered electron (BSE) image of UCAMM DC06-07-18-b displaying the two zones imaged with AFMIR (scales: A, $20 \times 10 \mu \mathrm{m}^{2}$; $\mathrm{B}, 10 \times 5 \mu \mathrm{m}^{2}$ ). The largest minerals identified are labelled (Sil: silicates ; FeS: iron sulphide; $\mathrm{AlO}_{X}$ : aluminium rxide). Right: sulphur, silicon, iron, magnesium, oxygen and aluminium X-ray maps.

UCAMM polyaromatic network. The band around $1710 \mathrm{~cm}^{-1}$ is attributed to a carbonyl bond. For comparison, the Fig. 3 (top) inset shows the far-field spectrum in the wavelength range covered by the current AFMIR setup.

Figure 3 (bottom) shows the AFMIR images recorded for the $1710 \mathrm{~cm}^{-1}$ (red) and $1600 \mathrm{~cm}^{-1}$ (green) bands, a (red-green) composite image, and the AFM topographical map. Intensity variability is observed at very small spatial scale (tens of $\mathrm{nm}$ ). Figure 4 shows the AFMIR intensity map of region A recorded at $1600 \mathrm{~cm}^{-1}$, with additional full-range AFMIR spectra (above and below the image frame) recorded for nine positions selected for their varying contrasts. A spectral deconvolution in two main peaks attributable to $\mathrm{C}=\mathrm{O}$ (green) and polyaromatic $\mathrm{C}=\mathrm{C}$ (red) components is shown, as well as the "classic" far-field spectrum covering the entire particle.

Positions 1, 2, and 3 are the best local representatives of the global spectrum (Fig. 4). Position 9 represents a spectral blank that corresponds to a mineral included in the UCAMM organic matter (bright spot on Fig. 2). The white isocontour delineates the height corresponding to $15 \%$ of the maximum height measured by the AFM tip, and outlines the overall particle shape. The AFMIR intensity ratio map at 1710 and $1600 \mathrm{~cm}^{-1}$ for region A is displayed in Fig. 4 (the ratio map for region B is presented in Appendix A.1). Both figures reveal for the first time heterogeneities at scales $(\sim 100 \mathrm{~nm})$ much lower than the IR diffraction limit. The histogram of the ratios extracted from the map of region A is displayed in Fig. 5 (bottom). Close to the central region of the map (on the bottom-left side of position 1), the organic matter is richer in $\mathrm{C}=\mathrm{C}$, that is, it is less oxidized than the region close to the mineral (positions 4, 7, 8). The BSE image shows some platelet-like structure in some regions of the grain. The central region, where the $\mathrm{C}=\mathrm{O} / \mathrm{C}=\mathrm{C}$ ratio is lower, exhibits a markedly different texture, less "platelet-like", as can be clearly seen on the BSE image. The oxidation variations may result from the formation processes of the organic matter from its precursors (potentially modified by parent-body processing) but may also be affected by the atmospheric entry of the particle. The AFMIR sampling spatial resolution appears to be mandatory for such small particles to focus on the least altered regions of the particle. The distribution of $\mathrm{O} / \mathrm{C}$ ratios probed by these modes can be evaluated using the ratio of $\mathrm{C}=\mathrm{O}$ to $\mathrm{C}=\mathrm{C}$ oscillator strengths
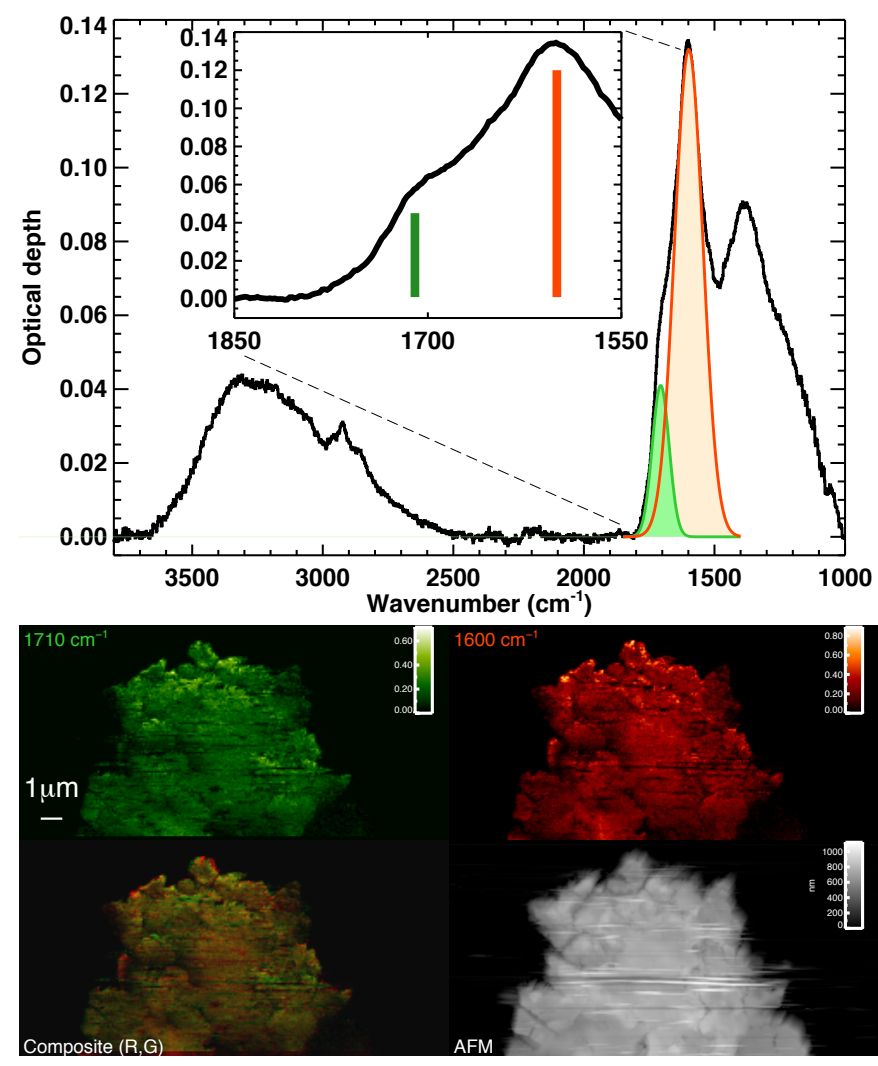

Fig. 3. Top: far-field spectrum (global spectrum acquired with a Nicolet Continuum II IR microscope on the SOLEIL SMIS beam line) of UCAMM DC16-07-18b. The inset displays the spectrum in the range covered by the laser for the AFMIR measurements. The band at $1710 \mathrm{~cm}^{-1}$ (green) is attributed to $\mathrm{C}=\mathrm{O}$, while the band at $1600 \mathrm{~cm}^{-1}$ (red) reflects the presence of $\mathrm{C}=\mathrm{C} / \mathrm{C}=\mathrm{N}$ bonds. Bottom: false colour maps showing the measured intensity maps of the bands at $1710 \mathrm{~cm}^{-1}$ (green) and $1600 \mathrm{~cm}^{-1}$ (red), and a composite image (red-green). Bottom right: topographical map recorded during the AFM measurements (greyscale).

derived previously and described in Dartois et al. (2013). Based on these oscillator strengths, and as the bands have similar full 

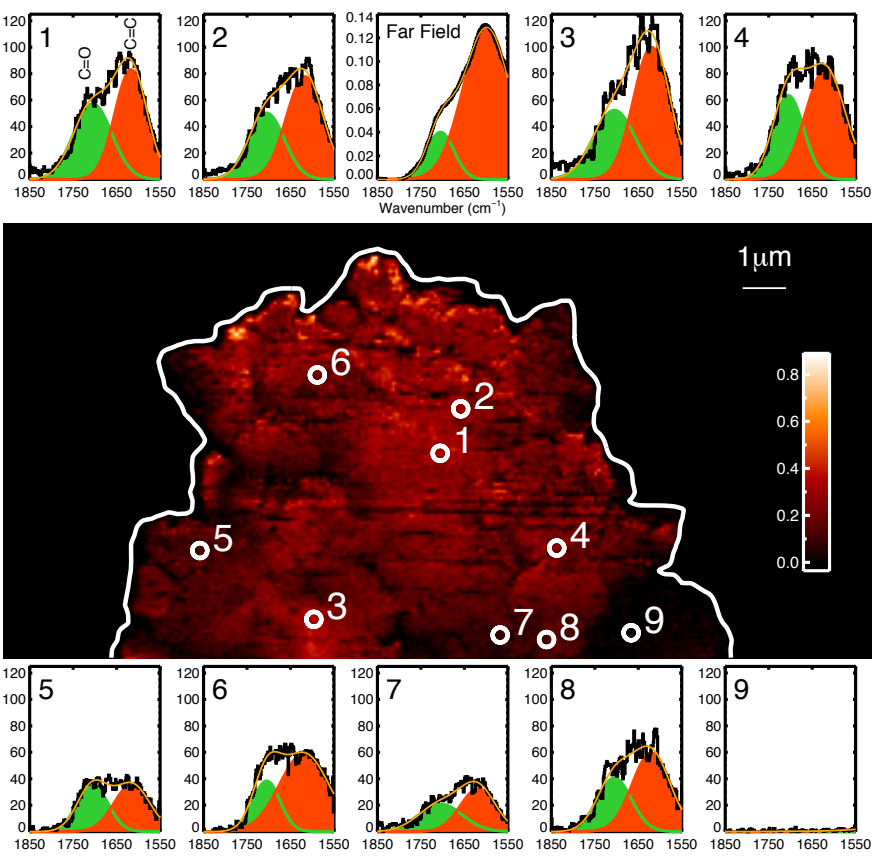

Fig. 4. AFMIR signal map at $1600 \mathrm{~cm}^{-1}$ of the region of interest "A" and overlaid selected positions (1-9) with full spectra surrounding the image. The far-field spectrum is shown in the same wavenumber range. The white isocontour delineates the height corresponding to $15 \%$ of the maximum height measured by the AFM tip. It delineates the AFM sample boundaries and outlines the overall particle shape.

width at half maximum (FWHM), we can multiply the measured map ratios by $\sim 0.07$ to evaluate an atomic $\mathrm{O} / \mathrm{C}$ ratio based on the $\mathrm{C}=\mathrm{O} / \mathrm{C}=\mathrm{C}$ bands. If the $\mathrm{O} / \mathrm{C}$ is dominated by these modes, the values displayed in the histogram of Fig. 5 span a range of $\mathrm{O} / \mathrm{C}$ ratios from about 0.015 to 0.10 from the first to the last quartile of the histogram.

Some $\mathrm{O}$ and $\mathrm{C}$ will be missing from the counting. The aliphatic carbons seen in the far-field spectrum represent a minor fraction of the $\mathrm{C}$ contribution and should not strongly affect the $\mathrm{O} / \mathrm{C}$ ratio. Indeed, the difference in intrinsic absorption strength between the $\mathrm{C}=\mathrm{C}$ and the $\mathrm{C}-\mathrm{H}$ is more than a factor of ten in favour of the $\mathrm{C}-\mathrm{H}$ stretches in general. In addition, the integral of the $\mathrm{C}-\mathrm{H}$ versus the $\mathrm{C}=\mathrm{C}$ bands is largely in favour of the $\mathrm{C}=\mathrm{C}$ contribution. All together these observations show that the $\mathrm{C}-\mathrm{H}$ contribution to the $\mathrm{C}$ budget is not dominant. Potential contributions from alcohol and ether groups are intrinsically more difficult to assess.

If one assumes that the $\mathrm{C}-\mathrm{H}$ stretching modes observed were only associated with alcohols and assuming the simplest alcohol with $\mathrm{CH}_{2}$ and $\mathrm{CH}_{3}$ (i.e. ethanol), the corresponding maximum contribution on the broad absorption around $3300 \mathrm{~cm}^{-1}$ from the $\mathrm{OH}$ would be about two times higher in optical depth than that of $\mathrm{C}-\mathrm{H}$. However, this value is clearly an upper limit as the assumption that all this absorption feature would be only associated to alcohols is certainly not realistic.

Moreover, no significant contribution from the alcohol to the $\mathrm{O}$ and $\mathrm{C}$ is expected based on the absence of a substantial contribution of the alcohol $\mathrm{C}-\mathrm{O}$ stretching mode in the $1200-1050 \mathrm{~cm}^{-1}$ range, which would be also at least twice time more intense than the $\mathrm{C}-\mathrm{H}$ ones for ethanol. Therefore, as discussed previously in Dartois et al. (2013), if the broad band at $3300 \mathrm{~cm}^{-1}$ may contain an eventual $\mathrm{O}-\mathrm{H}$ contribution, it is rather likely to be dominated by the $\mathrm{N}-\mathrm{H}$ chemical speciation
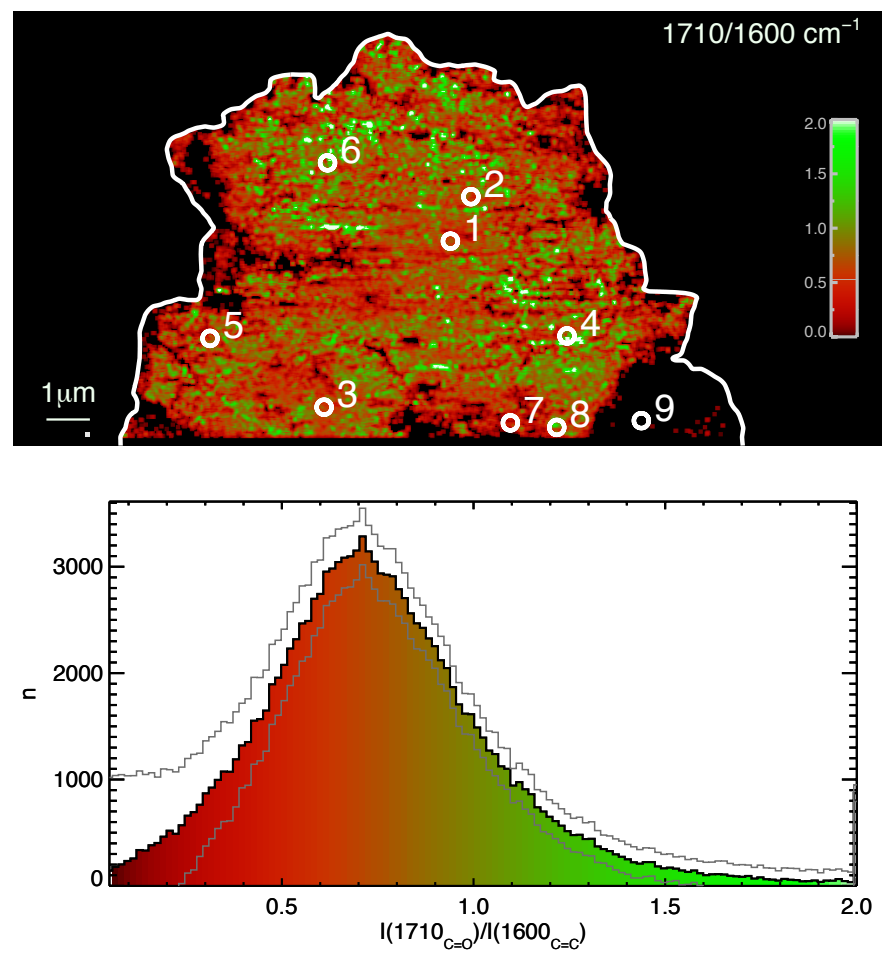

Fig. 5. Top: enhanced contrast palette image of the ratio of the AFMIR intensity maps at 1710 and $1600 \mathrm{~cm}^{-1}$ of region of interest "A". The white contour delineates the AFM sample boundaries as defined by the AFM. The white circles denote the locations where full spectra were acquired. Bottom: histogram of the ratio of the AFMIR maps at 1710 and $1600 \mathrm{~cm}^{-1}$ for the whole region of interest " $\mathrm{A}$ ".

of this N-rich organic matter constitutive of UCAMMs. The presence of ether is more difficult to measure, as its unique contribution is in the $1270-1100 \mathrm{~cm}^{-1}$ range. Most materials with a strong ether contribution show a peak in the 1270$1200 \mathrm{~cm}^{-1}$ region, whereas in the UCAMMs, the observed band profile peaks at much higher wavenumbers (around $1370 \mathrm{~cm}^{-1}$ ). There is thus no clear signature of ether in the spectra of UCAMMs studied in this work, nor is there, to our knowledge, a substantial contribution of ether in the STXM spectra of the other UCAMM samples studied by Yabuta et al. (2017), for example. The ratio presented in Fig. 5 provides a quantitative estimate of the evolution, at the AFMIR probed scale, of the oxygen involved in $\mathrm{C}=\mathrm{O}$ structures in relation to the aromatic macromolecular network, delineating structural changes at small scales.

\section{2. $D C 16-14-309-a$}

A correlative microscopy mosaic of analyses of the first DC1614-309 UCAMM microtome slice (DC16-14-309-a) is shown in Fig 6. The images were recorded at 1710,1600 , and $1460 \mathrm{~cm}^{-1}$ aiming at revealing the spatial distribution of the $\mathrm{C}=\mathrm{O}, \mathrm{C}=\mathrm{C}$, and $\mathrm{CH}_{2}$ vibrational modes. The far-field spectrum recorded after the AFMIR experiments shows that the $1460 \mathrm{~cm}^{-1}$ map is dominated by a broad absorption extending down to $1200 \mathrm{~cm}^{-1}$ and thus does not properly sample the $\mathrm{CH}_{2}$ mode in this case. The AFMIR composite image (overlay of the three channels) is therefore mainly purple (i.e. dominated by the combination of red, $1600 \mathrm{~cm}^{-1}$, and blue, $1460 \mathrm{~cm}^{-1}$, that seem to sample the same distribution). Furthermore, the regions where the carbonyl is locally higher appear greener. 

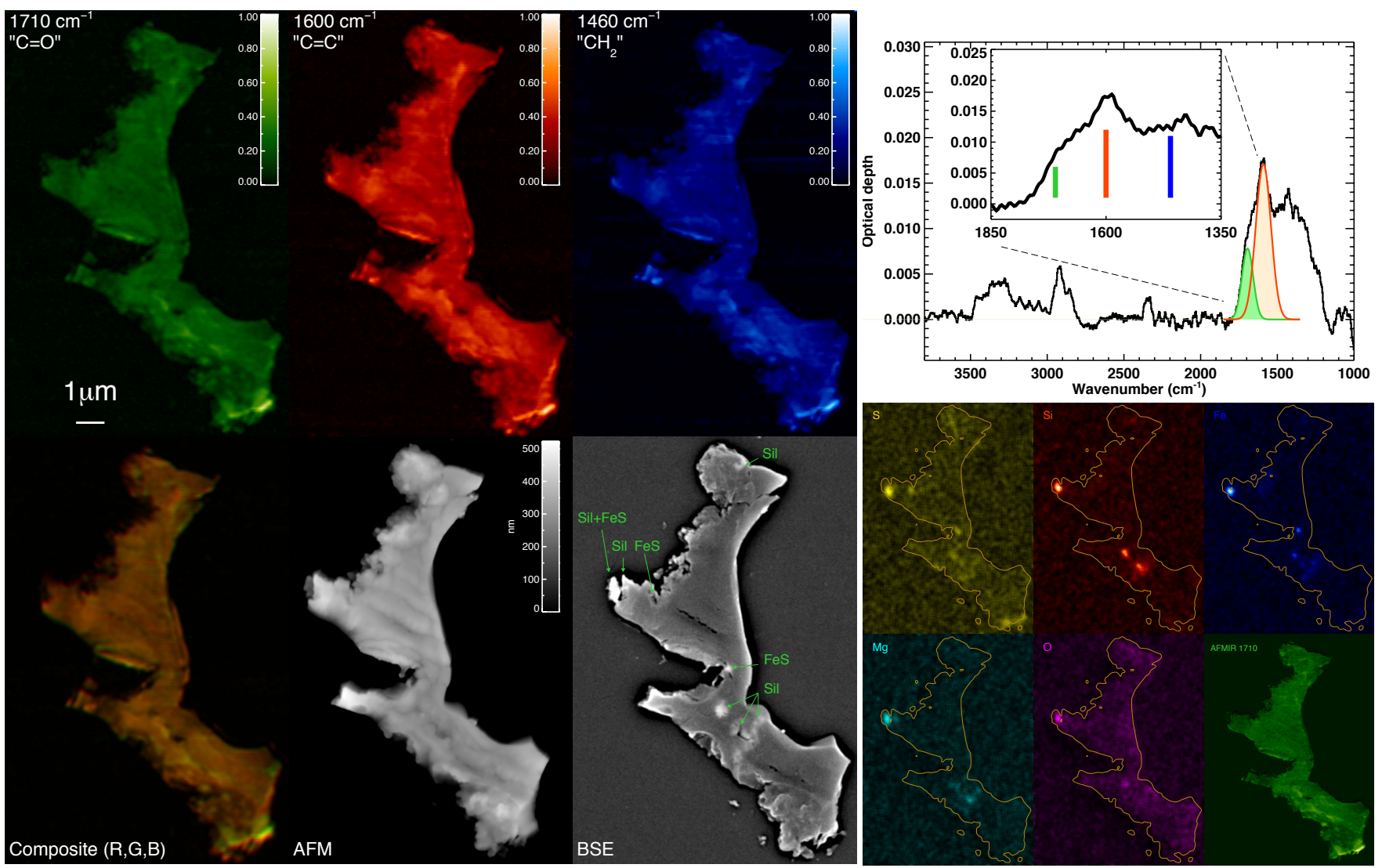

Fig. 6. Left: DC16-14-309-a AFMIR maps recorded at $1710 \mathrm{~cm}^{-1}$ (green), $1600 \mathrm{~cm}^{-1}$ (red), and $1460 \mathrm{~cm}^{-1}$ (blue), composite (red, green, blue) image of the three maps, and AFM height and back-scattered electron (BSE) image of UCAMM DC16-14-309-a. Top right: far-field spectrum. Vertical lines in the inset indicate the laser settings used to map the sample. Bottom right: sulphur, silicon, iron, magnesium, and oxygen X-ray maps. The largest minerals identified are labelled (Sil: silicates; FeS: iron sulphide). We note that the very bottom-right area seen in S without counterpart in Fe probably corresponds to elemental sulphur from the sample preparation. The AFMIR $1710 \mathrm{~cm}^{-1}$ map is shown at the same scale.

The X-ray maps identify the location of minerals that correspond to loss of signal in the AFMIR maps and bright spots in the BSE image. The intensity ratio map of $1710 \mathrm{~cm}^{-1}$ (targeting $\mathrm{C}=\mathrm{O}$ ) and $1600 \mathrm{~cm}^{-1}$ (targeting $\mathrm{C}=\mathrm{C} / \mathrm{C}=\mathrm{N}$ modes) AFMIR maps of DC16-14-309-a is displayed in Fig. 7, together with four spectral scan maps recorded on different line positions on the sample ( $\mathrm{S} 1$ to $\mathrm{S} 4$, blue squares). As discussed previously, an evaluation of an atomic $\mathrm{O} / \mathrm{C}$ ratio based on the $\mathrm{C}=\mathrm{O} / \mathrm{C}=\mathrm{C}$ bands ratio can be performed by multiplying the measured map ratios by $\sim 0.07$.

The sizes of the largest minerals are much smaller in this sample than those observed in DC06-07-18-b and no systematic variation of the composition of the organic matter in contact with the mineral was seen. The bright spot on the bottom right rim, readily identified on the last $\mathrm{S} 1$ spectrum Fig. 6, is different from the bulk UCAMM spectra. Such very-small-scale variations associated with such tiny grain components escape detection on the far-field spectrum as they are blended with the bulk UCAMM volume.

\section{3. $D C 16-14-309-b$}

A multi-technique mosaic of analyses of another DC16-14-309 UCAMM microtome slice (DC16-14-309-b) is shown in Fig 8. The images were recorded at the same laser wavenumbers as for the previous sample, except for the $\mathrm{CH}_{2}$ vibrational mode map, which was tuned to $1470 \mathrm{~cm}^{-1}$ (after noting that the AFMIR signal was high at this wavenumber). There is a clear spatial anticorrelation between the $1710 \mathrm{~cm}^{-1}$ (green) and $1600 \mathrm{~cm}^{-1}$ (red) maps on one side, and the $1470 \mathrm{~cm}^{-1}$ (blue) map on the other side. The $1470 \mathrm{~cm}^{-1}$ map clearly identifies a spurious feature (bottom right) that may result from contamination from the sample-preparation procedure. This observation strengthens the importance of spatially resolved spectral measurements at small scales. In order to identify the far-field component that may include this feature, we recorded two spectra, at the limit of what classical spectroscopy can provide, using two different apertures: one covering the spurious part identified with AFMIR measurements and one not (see top right of Fig 8).

The upper spectrum is the noisier and corresponds to the almost pure UCAMM spectrum, whereas the lower spectrum includes the spurious feature. If unresolved, the global spectrum would seem to show a globally aliphatic rich spectrum for the particle. By contrast, the AFMIR maps allow for us to discard the corresponding region of the fragment and to discriminate sub-micron contaminated regions from genuine ones.

The intensity ratio map of $1710 \mathrm{~cm}^{-1}$ (targeting $\mathrm{C}=\mathrm{O}$ ) and $1600 \mathrm{~cm}^{-1}$ (targeting $\mathrm{C}=\mathrm{C} / \mathrm{C}=\mathrm{N}$ modes) AFMIR maps of DC1614-309-b is displayed in Fig. 9, as well as four spectral maps (S1 to S4) recorded at different line positions on the sample. This map reveals the UCAMM fine-scale composition and is unaffected by the spurious feature that is suppressed as it 

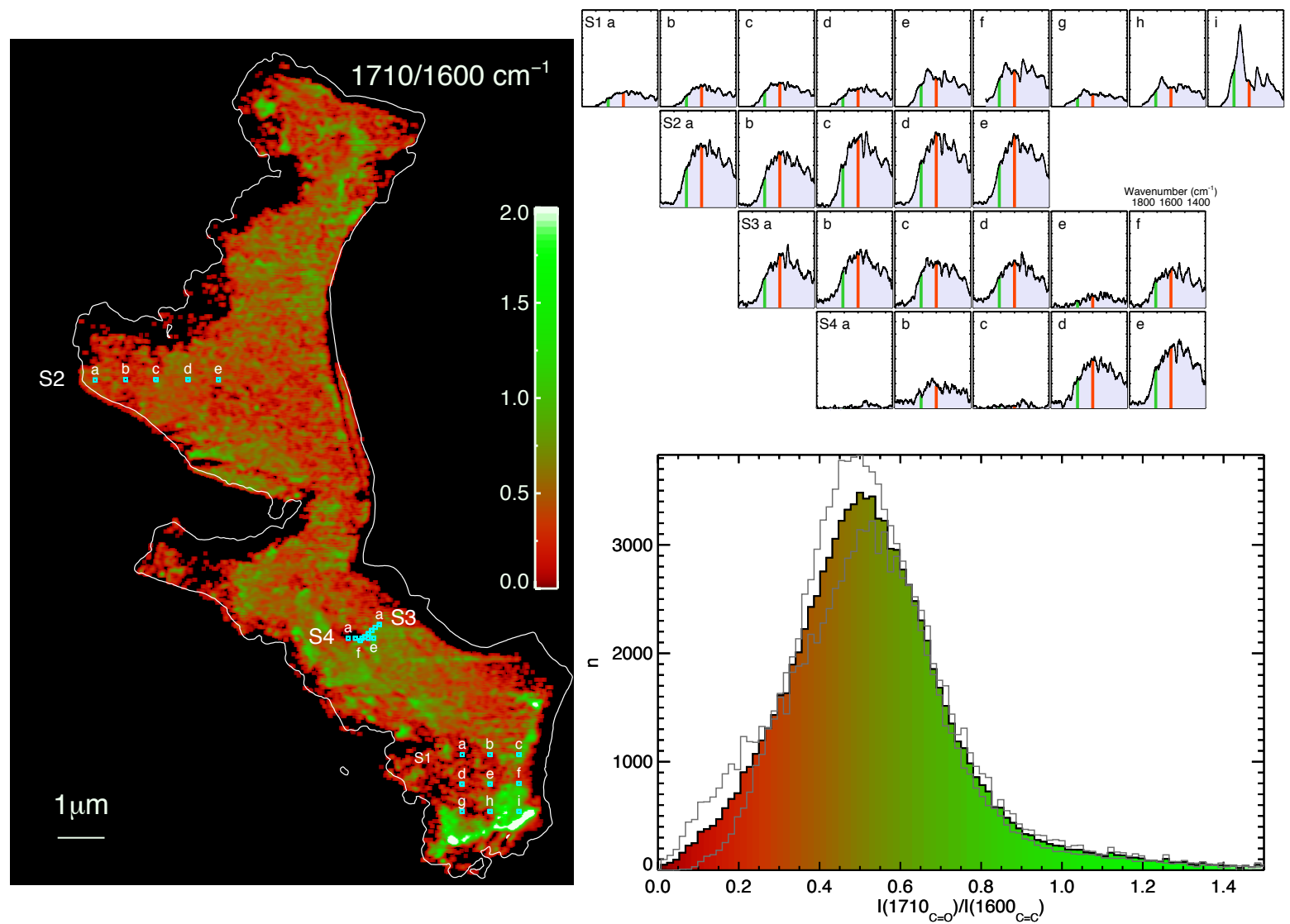

Fig. 7. DC16-14-309-a. Left: ratio of the AFMIR maps at 1710 and $1600 \mathrm{~cm}^{-1}$. The white isocontour delineates the height corresponding to $5 \%$ of the maximum height measured by the AFM tip. Top right: AFMIR spectral maps in the $1900-1350 \mathrm{~cm}^{-1}$ range (labelled S1 to S4, performed in selected locations shown on the map). Vertical lines mark the 1710 and $1600 \mathrm{~cm}^{-1}$ wavenumber positions. Bottom right: histogram of the ratio of the AFMIR maps at 1710 and $1600 \mathrm{~cm}^{-1}$.
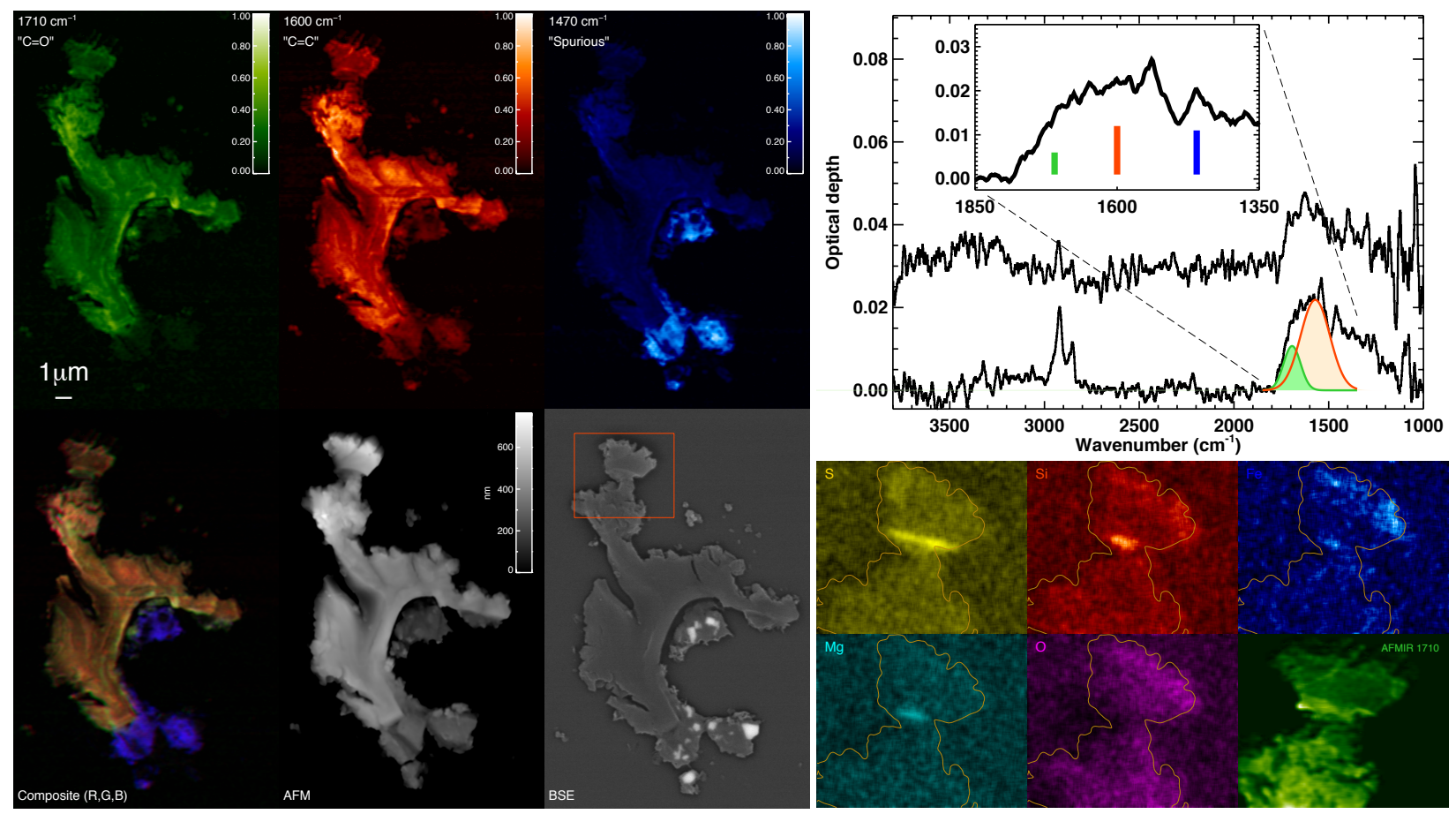

Fig. 8. Left: DC16-14-309-b AFMIR maps recorded at $1710 \mathrm{~cm}^{-1}$ (green), $1600 \mathrm{~cm}^{-1}$ (red), and $1470 \mathrm{~cm}^{-1}$ (blue), composite (red, green blue) image of the three maps, AFM height, and back-scattered electrons (BSE) image (the bottom-right bright spots are associated with a contamination, with recorded spectra shown in Fig. 9). The orange square delineates the region analysed by EDX. Top right: far-field spectrum. Vertical lines in the inset indicate the laser settings used to map the sample. Bottom right: sulphur, silicon, iron, magnesium and oxygen X-ray maps. The bright spot in $\mathrm{Si}, \mathrm{Mg}$ and $\mathrm{Fe}$ corresponds to silicates. The more extended sulphur spots probably include some elemental sulphur from the sample preparation. The AFMIR $1710 \mathrm{~cm}^{-1}$ map is shown at the same scale. 

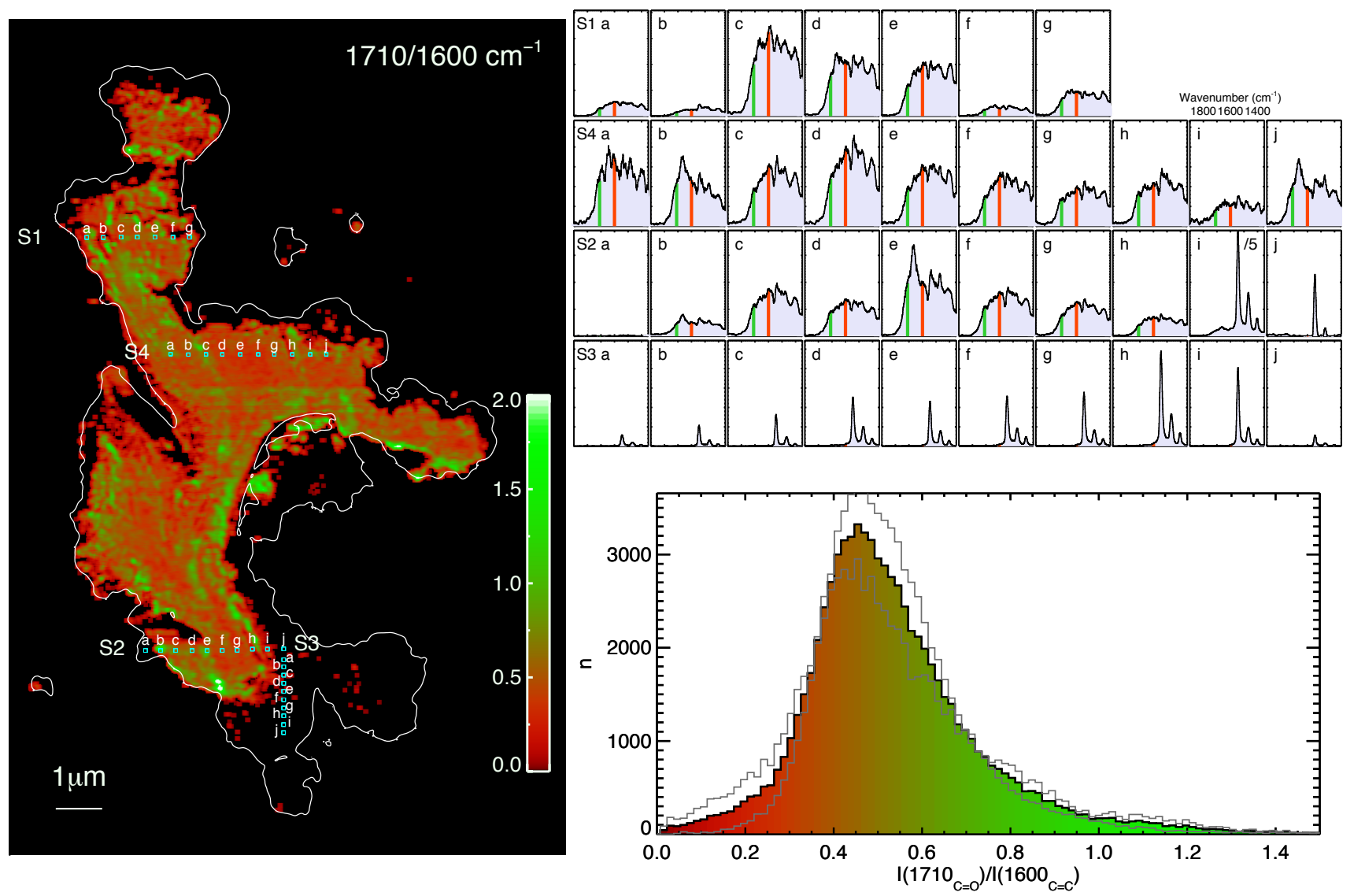

Fig. 9. DC16-14-309-b. Left: ratio of the AFMIR maps at 1710 and $1600 \mathrm{~cm}^{-1}$. The white isocontour delineates the height corresponding to $5 \%$ of the maximum height measured by the AFM tip. Top right: AFMIR spectral maps (labelled S1 to S4, performed in selected locations shown on the map) in the $1900-1350 \mathrm{~cm}^{-1}$ range. Vertical lines mark the 1710 and $1600 \mathrm{~cm}^{-1}$ wavenumber positions. Bottom right: histogram of the ratio of the AFMIR maps at 1710 and $1600 \mathrm{~cm}^{-1}$.

possesses no contribution at $1710 \mathrm{~cm}^{-1}$. The spurious feature, hardly seen in the far-field spectra, is readily identified in the S3 spectral scans and possesses characteristic narrow bands forming a triple-peak absorption set. These bands correspond to the signature of zinc stearate and the presence of zinc in the bright spots in the BSE image is confirmed by SEM/EDX. This compound is commonly used in paints, plastics, and the rubber industry, and this contamination at the micron level is thought to have happened during the sample preparation of this slice.

The 1710 and $1600 \mathrm{~cm}^{-1}$ ratio maps of both of the DC1614-309 UCAMM fragments show patches with higher ratios, with spatial distribution variations at the sub-micron scale. Similar patches are observed on one organic component analysed by STXM (e.g. Charon et al. 2017) for UCAMMs. These patches appear to be associated with alkene $\mathrm{C}=\mathrm{C}$ and ketone components in the STXM X-ray-absorption near-edge-structure spectral images (see Fig. 3 Charon et al. 2017). The potential presence of an alkene $\mathrm{C}=\mathrm{C}$ component (around $1640 \mathrm{~cm}^{-1}$ ) associated with a ketone absorption in the IR would enhance the $1710 / 1600 \mathrm{~cm}^{-1}$ ratio in the AFMIR measured spectra. This would be the case for spectra such as the S1-i in Fig. 7 and S4-b, S4-j, and S2-e in Fig. 9. The measurements performed did not include a map at $1640 \mathrm{~cm}^{-1}$, which would allow for a direct olefinic to aromatic $\mathrm{C}=\mathrm{C}$ ratio estimate to be made. Given the sub-micron sizes of the patches observed by STXM this implies that the AFMIR measurements will allow for a spectral identification of this component, which would otherwise escape detection in a classical diffraction-limited IR analysis with typical sampling of several microns size.

\section{Conclusions}

Contrary to minerals, which can be imaged at very high spatial resolution with electronic techniques (TEM, SEM) and whose composition can be characterised by for example X-ray spectroscopy, variations in the nature of the chemical bonds of organic matter are much more complex and therefore difficult to investigate. Infrared techniques, such as FTIR, provide such characterisation (and as a non-destructive technique, without modifying the sample), but are often limited in spatial resolution by the diffraction imposed by the wavelength of interest. AFMIR measurements are able to image chemical functional groups at very fine scales that are relevant to the study of interplanetary dust particles such as micrometeorites, with typical inclusion sizes ranging from tens to hundreds of nanometers. The AFMIR measurements presented in this study open the way to exploring quantitative functional groups maps on extraterrestrial matter. Unprecedented maps of the functional groups of interplanetary organic matter are revealed at spatial resolutions of a few tens to hundreds of nanometers. In the range explored in this study (limited to $1900-1350 \mathrm{~cm}^{-1}$ at this time), we observed variations of the carbonyl to $\mathrm{C}=\mathrm{C}$ modes in one UCAMM sample, giving access to the inhomogeneities of the oxygen content of carbonyl within the organic matter. Imaging the degree of organic-matter oxidation is a difficult task when using techniques 
measuring only the elemental atomic ratios. Nonetheless, the work presented here shows that AFMIR measurements can provide such mapping at small scales. The second UCAMM sample shows patches of a few tens to hundred of nanometers, similar to the dusty patches observed for the organic components analysed by STXM in the same UCAMM (Charon et al. 2017). The corresponding spectrum would be consistent with a locally enhanced olefinic $\mathrm{C}=\mathrm{C}$ contribution, embedded in an organic matrix with a different composition dominating the large-scale (far field) IR spectrum. There is room for improvement of the AFMIR measurements on such complex extraterrestrial material presented in this study. The next steps for a comprehensive view of the chemical composition and nanoscale description of UCAMM organic matter will consist in further investigation of the relationship between the structures seen in STXM and AFMIR, and the development of hyperspectral techniques allowing us to access the entire IR spectrum at the same sensitivity level as that probed in this study.

Acknowledgements. Experiments were performed on the NanoIR 2-s microscope installed on the SMIS3 beamline, and using the chemistry support lab at SOLEIL Synchrotron, France. This work was supported by the French Agence Nationale de la Recherche (COMETOR, ANR-18-CE31-0011) as well as INSU (PNP, PCMI), IN2P3, CNES, DIM-ACAV (Région Île de France), CNRS, the "Déf Origines" from the MITI (CNRS) and Universite Paris-Sud. This work is part of the JWST emblematic project from the LABEX-P2IO. We are grateful to the French and Italian polar institutes IPEV and PNRA, for their financial and logistic support of the micrometeorites collection at the vicinity of the CONCORDIA station (Dome C).

\section{References}

Alleon, J., Bernard, S., Le Guillou, C., et al. 2017, Sci. Rep., 7, 1508

Augé, B., Dartois, E., Engrand, C., et al. 2016, A\&A, 592, A99

Bardin, N., Slodzian, G., Wu, T.-D., et al. 2014, Lunar Planet. Sci. Conf., 45, 2647

Charon, E., Engrand, C., Benzerara, K., et al. 2017, Lunar Planet. Sci. Conf., 48, 2085

Dartois, E., Engrand, C., Brunetto, R., et al. 2013, Icarus, 224, 243

Dartois, E., Chabot, M., Pino, T., et al. 2017, A\&A, 599, A130

Dartois, E., Engrand, C., Duprat, J., et al. 2018, A\&A, 609, A65

Dazzi, A., \& Prater, C. 2017, Chem. Rev. 117, 5146

Dazzi, A., Prater, C. B., Hu, Q., et al. 2012, Appl. Spectr., 66, 1365

Dobrica, E., Engrand, C., Duprat, J., \& Gounelle, M. 2010, Meteorit. Planet. Sci. Suppl., 73, 5213

Dominguez, G., McLeod, A. S., Gainsforth, Z., et al. 2014, Nat. Commun., 5, 5445

Duprat, J., Engrand, C., Maurette, M., et al. 2007, Adv. Space Res., 39, 605

Duprat, J., Dobrică, E., Engrand, C., et al. 2010, Science, 328, 742

Engrand, C., \& Maurette, M. 1998, Meteorit. Planet. Sci., 33, 565

Kebukawa, Y., Nakashima, S., Ishikawa, M., et al. 2010, Meteorit. Planet. Sci., 45, 394

Lu, F., \& Belkin, M. A. 2011, Opt. Exp., 19, 19942

Lu, F., Jin, M., \& Belkin, M. A. 2014, Nat. Photon., 8, 307

Mathurin, J., Pancani, E., Deniset-Besseau, A., et al. 2018, Analyst, 143, 5940

Nakamura, T., Noguchi, T., Ozono, Y., Osawa, T., \& Nagao, K. 2005, Meteorit. Planet. Sci. Suppl., 40, 5046

Poppe, A. R. 2016, Icarus, 264, 369

Ramer, G., Aksyuk, V., Centrone, A. 2017, Anal. Chem., 89, 13524

Taylor, S., Alexander, C. M. O., \& Wengert, S. 2008, Lunar Planet. Sci. Conf., 39,1628

van Ginneken, M., Folco, L., Cordier, C., \& Rochette, P. 2012, Meteorit. Planet. Sci., 47, 228

Yabuta, H., Itoh, S., Noguchi, T., et al. 2012, Lunar Planet. Sci. Conf., 43, 2239

Yabuta, H., Noguchi, T., Itoh, S., et al. 2017, Geochim. Cosmochim. Acta, 214, 172

Yesiltas, M., \& Kebukawa, Y. 2016, Meteorit. Planet. Sci., 51, 584

\section{Appendix A: DC06-07-18-b}

Same analysis as presented in the article for region $\mathrm{A}$, but for region B (see Fig. 2) spanning $5 \times 10 \mu \mathrm{m}$.
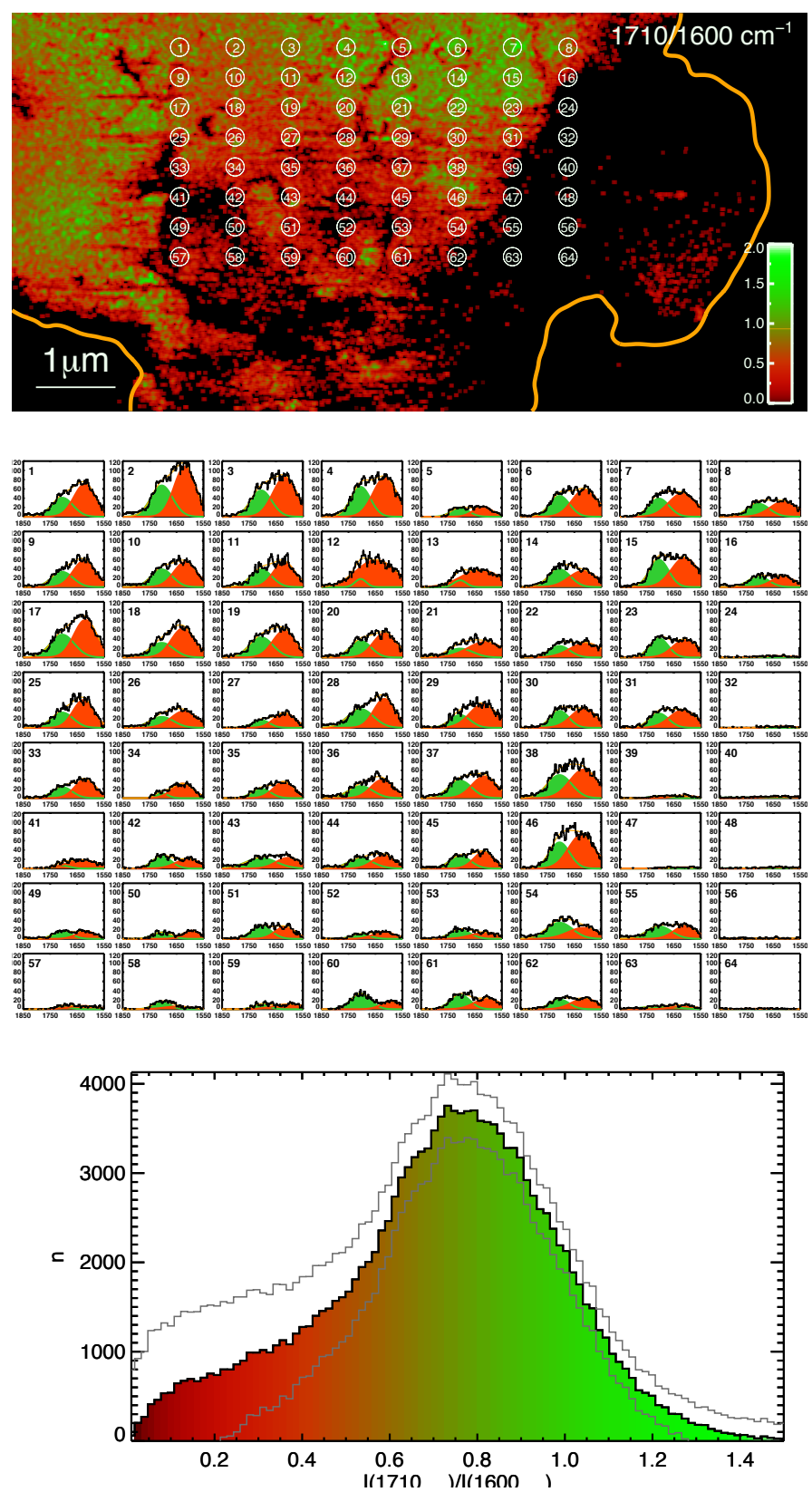

Fig. A.1. Top: DC06-07-18-b, ratio of the AFMIR maps at 1710 and $1600 \mathrm{~cm}^{-1}$ of the region of interest $\mathrm{B}$. The orange contour delineates the AFM sample boundaries. Positions for the individual spectra presented in the middle panel recorded on a $8 \times 8$ equally sampled map are indicated on top of the ratio map. Middle: individual AFMIR spectra in the $1850-1550 \mathrm{~cm}^{-1}$ range recorded on an evenly spaced $8 \times 8$ map, with $0.7 \mu \mathrm{m}$ spacing on the $x$-axis and $0.38 \mu \mathrm{m}$ spacing on the $y$-axis. The spectra are deconvolved into two main bands corresponding to the carbonyl $(\mathrm{C}=\mathrm{O})$ dominating the $1710 \mathrm{~cm}^{-1}$, and contribution from the carbon backbone contributing to the $1600 \mathrm{~cm}^{-1}$ map. Bottom: histogram of the ratio of the AFMIR maps at 1710 and $1600 \mathrm{~cm}^{-1}$ of region of interest $\mathrm{B}$. 